\title{
Screening of Key Genes Associated with Ischemic Stroke via Microarray Data
}

\author{
Jianmin Wang, Dongliang Zhou, Hongwei Qin, Ying Xu, Ying Guan, \\ Weidong Zang
}

\begin{abstract}
Objective: To promote understandings about the pathogenesis of ischemic stroke (IS) through mining key genes, functions and pathways with microarray technology. Methods: Differentially expressed genes (DEGs) in blood between patients with IS and healthy people were screened out through comparing microarray data obtained from Gene Expression Omnibus. Overrepresented functions in DEGs were revealed by Gene Ontology (GO) enrichment analysis. Interaction network was constructed for the top 24 DEGs with information from Human Protein Reference Database (HPRD). Relevant microRNAs (miRNAs) were retrieved from three databases: TargetScan, miRBase and miRanda. Results: A total of 503 DEGs were obtained. Functional enrichment analysis showed that immune response, signaling pathways and apoptosis were significantly over-represented. Six key genes with big degree, betweenness and clustering coefficient were then revealed, which might play important roles in the development of IS. In addition, 57 differentially expressed miRNAs targeting the 6 genes were retrieved. Conclusions: Our study provides insights into the pathogenesis of IS and potential targets to treat the disease.
\end{abstract}

RÉSUMÉ: Dépistage de gènes clés associés à l'accident vasculaire cérébral ischémique au moyen de données obtenues par la technique des biopuces. Objectif : Le but de l'étude était de favoriser la compréhension de la pathogenèse de l'accident vasculaire cérébral ischémique (AVCI) en explorant des gènes, des fonctions et des voies de signalisation clés au moyen de la technique des biopuces. Méthode : Des gènes différentiellement exprimés (GDE) dans le sang de patients atteints d'un AVCI et de sujets sains ont été étudiés en comparant les données acquises par la technique des micropuces obtenues de Gene Expression Omnibus. Les fonctions surreprésentées dans les GDE ont été identifiées par le test d'enrichissement basé sur le Gene Ontology. Un réseau d'interactions a été construit pour les 24 premiers GDE au moyen d'informations obtenues de la Human Protein Reference Database. Les micro-ARN pertinents ont été obtenus de trois bases de données : TargetScan, miRBase et miRanda. Résultats : Nous avons obtenu 503 GDE en tout. L'analyse d'enrichissement fonctionnel a montré que la réponse immunitaire, les voies de signalisation et l'apoptose étaient surrepésentées de façon significative. Six gènes clés ayant un coefficient élevé d'intermédiarité et de clustering ont ensuite été identifiés. Ils pourraient jouer des rôles importants dans la genèse de l'AVCI. De plus, nous avons identifié 57 micro-ARN différentiellement exprimés ciblant les 6 gènes. Conclusions : Notre étude fournit des informations sur la pathogenèse de l'AVCI et des cibles potentielles de traitement de la maladie.

Can J Neurol Sci. 2013; 40: 864-869

Ischemic stroke (IS) is one of the major diseases imposing serious harm to human health. It is the leading causes of longterm disability and has mortality rates just second only to cancer $^{1}$.

People have been trying to disclose the pathogenesis. Tang et al analyzed the gene expression in blood after IS using oligonucleotide microarrays ${ }^{2}$. Proteomic analysis is also widely adopted ${ }^{3,4}$. Inflammation has been confirmed to play an important role in the pathogenesis of IS $^{5}$. Cytokines, including both pro-inflammatory and anti-inflammatory cytokines, are the focus of research ${ }^{6,7}$. Some cytokines show potential in diagnosis and prognosis, such as interleukin (IL) $-6^{8}$. However, the mechanisms are rather complicated and many questions remain to be answered. Among the various techniques, microarray technology is a powerful tool which enables us to globally investigate alterations in gene expression.

MicroRNAs (miRNAs), a class of non-transcribed small RNA molecules, are found to participate in a range of physiological and pathological processes, such as brain development $^{9}$ and tumorigenesis ${ }^{10,11}$. MicroiRNAs play critical roles in a variety of physiological and pathological processes as regulators of gene expression. Alterations in miRNA expression have been reported in $\mathrm{IS}^{12}$. The regulatory relationships between miRNAs and target genes are studied to deepen understanding about mechanisms involved in the development of IS.

The Gene Expression Omnibus (GEO) repository was established by National Center for Biotechnology Information (NCBI) which is currently the largest and fully public gene expression resource ${ }^{13}$. The database archives high-throughput molecular abundance data and predominantly gene expression data, and supports MIAME (Minimum Information About a

From the Department of Neurology (JW, DZ, HQ, YX, YG), Baoshan Branch of Huashan Hospital, Fudan University; FengHe (ShangHai) Information Technology Co. Ltd. (WZ), Shanghai, China.

Received April 19, 2013. Final Revisions Submitted June 27, 2013. Correspondence to: Jianmin Wang, No. 1999 Changjiang West Road, Shanghai 200431, China. Email: wangjianmin5588885@hotmail.com. 
Microarray Experiment)-compliant data submissions ${ }^{14}$. The primary roles of GEO are data archiving, functioning as a hub for data deposit and retrieval ${ }^{15,16}$.

In the present study, gene expression profiles for IS samples and healthy samples were downloaded from GEO and compared to screen out key genes. Portuguese and Spanish stroke cases and controls were ascertained and collected as described previously for Portuguese samples ${ }^{17}$, for Spanish cases ${ }^{18}$, and for Spanish controls $^{19}$. All participants were adults and Caucasian. Spanish patients were classified into causative subtypes according to the Trial of Org 10172 in Acute Stroke Treatment classification ${ }^{20}$. Ischemic stroke patients were required to have suffered only one stroke episode, at least six months before the blood collection, and controls could not have a family history of stroke. Participants with severe anemia or active allergies were also excluded $^{21}$. Functional enrichment analysis and interaction network analysis were then performed to learn their roles in development of IS. The miRNAs of the key genes were retrieved, which was a beneficial supplement in understanding the mechanisms.

\section{Materials ANd Methods}

\section{Microarray data}

Microarray data set GSE22255 21 was downloaded from GEO (http://www.ncbi.nlm.nih.gov/geo/), including 20 IS samples and 20 sex- and age-matched healthy controls. DNA was extracted from peripheral blood monocytes and raw data was collected with Affymetrix Human Genome U133 Plus 2.0 Array. Pre-treatment, background correction and RMA normalization were conducted with package Affy of R for the raw data, which generated 54675 probes in total.

\section{Screening of differentially expressed genes (DEGs)}

The expression levels for each probe between IS samples and controls were compared with the original method ${ }^{21}$. Analysis of variance was used to identify the DEGs among IS samples and controls, taking into account known experimental (type, sex, and age) and study design (geographic origin and scan date) covariates ( $\mathrm{P}$ value). To control the false positive rate and screen out IS-associated genes, fold change of $>1.2$ and $\mathrm{Q}^{22}$ of $<0.05$ were set as the cut-offs.

\section{Functional enrichment analysis}

To identify significantly altered biological functions in IS, Gene Ontology (GO, http://www.geneontology.org/) ${ }^{23}$ enrichment analysis was performed for the DEGs. p value of < 0.05 was chosen as the threshold.

\section{Construction of miRNAs-target genes database}

MicroRNAs play an important role in regulation of gene expression and are thus involved in a variety of physiological and pathological processes. Regulatory information about miRNAs and target genes was gathered from three databases: TargetScan ${ }^{24}$, miRBase ${ }^{25}$ and miRanda ${ }^{26}$. Regulatory relationships observed in at least two databases were selected out and 533 miRNAs, 17734 target genes and 498812 interactions were acquired.

\section{Results}

\section{Differentially expressed genes}

A total of 709 probes meeting the above mentioned criteria were selected out. Those linked with more than one gene or no gene were removed and 503 DEGs were acquired. The top 30 probes with maximum alteration were chosen and underwent similar filtering, generating 24 DEGs, which might play a key roles in the development of IS.

\section{Functional enrichment analysis results}

GO enrichment analysis was performed for all the DEGs and FDR of $<0.05$ was chosen as the cut-off to screen out significantly disturbed biological functions in IS. The results are shown in Table 1. Defense response, signaling pathway and apoptosis were major terms significantly enriched in the DEGs.

\section{Interaction network}

The protein-protein interaction network was constructed for the top 24 DEGs using information from Human Protein Reference Database to further characterize their roles in pathogenesis of IS. The process was as follows: (1) selfinteractions were removed and 9641 genes with 36891 interactions were obtained; (2) the overlapping genes between the above group and Affymetrix Human Genome U133 Plus 2.0 Array were selected out and then a network containing 9239 genes and 71748 interactions were generated; (3) the 24 DEGs were entered into the network and 16 of them had annotations with 111 interactions; (4) finally the network was visualized with Cytoscape (Figure 1).

From Figure 1, we found that 6 genes were located in the center of networks: TNF, neural cell adhesion molecule 1

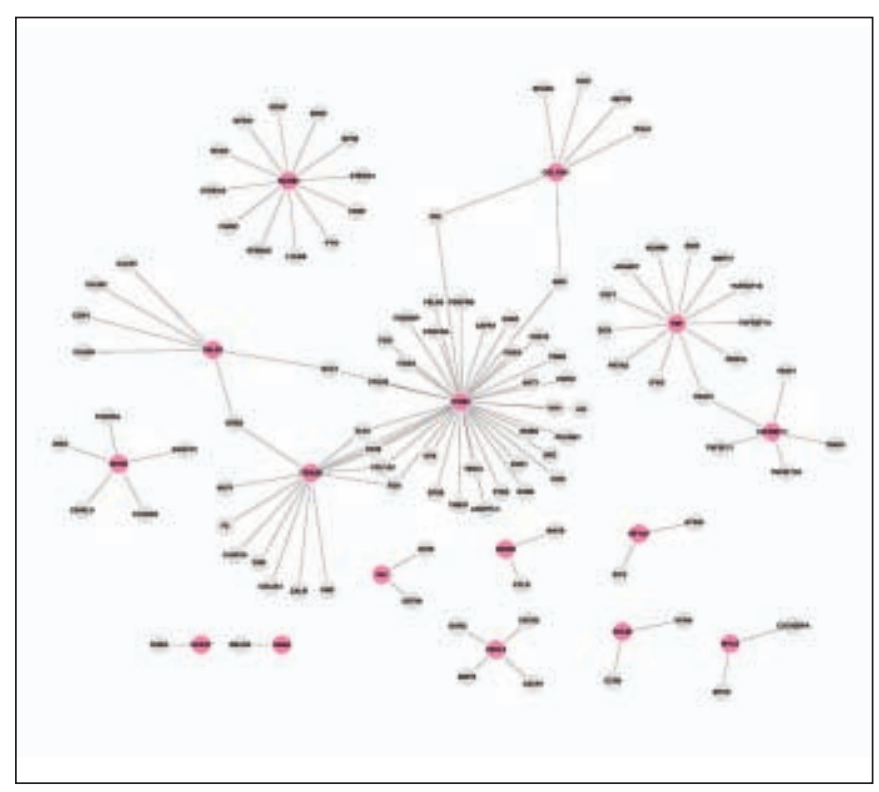

Figure 1: Interaction networks for 16 DEGs generated in this study. The 16 DEGs are in red while other interacting proteins are in gray. 
Table 1: GO enrichment analysis result for all the DEGs

\begin{tabular}{|c|c|c|c|}
\hline No. & Terms & No. of DEGs & $P$ value \\
\hline GO:0006950 & response to stress & 126 & $1.68397 \mathrm{E}-05$ \\
\hline GO:0002376 & immune system process & 86 & $1.78536 \mathrm{E}-05$ \\
\hline GO:0006952 & defense response & 60 & 0.000105993 \\
\hline GO:0045087 & innate immune response & 37 & 0.000105993 \\
\hline GO:0006955 & immune response & 58 & 0.000249827 \\
\hline GO:0030168 & platelet activation & 24 & 0.000291928 \\
\hline GO:0051707 & response to other organism & 36 & 0.000830566 \\
\hline GO:0009607 & response to biotic stimulus & 37 & 0.001008894 \\
\hline GO:0007596 & blood coagulation & 35 & 0.001112934 \\
\hline GO:0007599 & hemostasis & 35 & 0.001112934 \\
\hline GO:0050817 & coagulation & 35 & 0.001112934 \\
\hline GO:0071345 & cellular response to cytokine stimulus & 24 & 0.001307617 \\
\hline GO:0009611 & response to wounding & 56 & 0.00150621 \\
\hline GO:0050878 & regulation of body fluid levels & 38 & 0.001531905 \\
\hline GO:0009615 & response to virus & 21 & 0.002527213 \\
\hline GO:0034097 & response to cytokine stimulus & 28 & 0.002527213 \\
\hline GO:0010033 & response to organic substance & 71 & 0.002527213 \\
\hline GO:0060337 & type I interferon-mediated signaling pathway & 11 & 0.003160633 \\
\hline GO:0071357 & cellular response to type I interferon & 11 & 0.003160633 \\
\hline GO:0034340 & response to type I interferon & 11 & 0.003453129 \\
\hline GO:0042060 & wound healing & 37 & 0.005808023 \\
\hline GO:0045639 & positive regulation of myeloid cell differentiation & 9 & 0.006560905 \\
\hline GO:0002682 & regulation of immune system process & 44 & 0.007063413 \\
\hline GO:0019221 & cytokine-mediated signaling pathway & 20 & 0.007063413 \\
\hline GO:0002576 & platelet degranulation & 11 & 0.007780112 \\
\hline GO:0032606 & type I interferon production & 9 & 0.008986186 \\
\hline GO:0070887 & cellular response to chemical stimulus & 59 & 0.009609044 \\
\hline GO:0071310 & cellular response to organic substance & 44 & 0.0248592 \\
\hline GO:0032479 & regulation of type I interferon production & 8 & 0.026920838 \\
\hline GO:0001775 & cell activation & 39 & 0.026920838 \\
\hline GO:0012501 & programmed cell death & 65 & 0.029002463 \\
\hline GO:0008219 & cell death & 70 & 0.029002463 \\
\hline GO:0032940 & secretion by cell & 35 & 0.029002463 \\
\hline GO:0016265 & death & 70 & 0.02900369 \\
\hline GO:0006936 & muscle contraction & 18 & 0.031980533 \\
\hline GO:0052548 & regulation of endopeptidase activity & 17 & 0.034801436 \\
\hline GO:0006915 & apoptosis & 64 & 0.034801436 \\
\hline GO:0042981 & regulation of apoptosis & 53 & 0.03826052 \\
\hline GO:0051704 & multi-organism process & 48 & 0.038788939 \\
\hline GO:0042787 & protein ubiquitination involved in ubiquitin-dependent protein catabolic process & 8 & 0.038788939 \\
\hline GO:0043065 & positive regulation of apoptosis & 32 & 0.038788939 \\
\hline GO:0043067 & regulation of programmed cell death & 53 & 0.041065343 \\
\hline GO:0042221 & response to chemical stimulus & 100 & 0.041065343 \\
\hline GO:0043068 & positive regulation of programmed cell death & 32 & 0.041065343 \\
\hline GO:0034341 & response to interferon-gamma & 11 & 0.041065343 \\
\hline GO:0052547 & regulation of peptidase activity & 17 & 0.041065343 \\
\hline GO:0071346 & cellular response to interferon-gamma & 10 & 0.046042896 \\
\hline
\end{tabular}

(NCAM1, CD56), integrin beta 3 (ITGB3, CD61), integrin alpha 2b (ITGA2B, CD41), collagen type XIII alpha 1 (COL13A1) and caldesmon 1 (CALD1). To further describe the characteristics of the six genes, their degree, betweenness and clustering coefficient were calculated and shown in Table 2. They had degree bigger than six as well as big betweenness, suggesting that they might mediate several pathways and were important players in biological functions. High clustering coefficient was also observed, indicating that they tended to form modules. These properties implied that they were key genes in the development of IS.

\section{Regulatory network between microRNAs and target genes}

Interactions between the six genes and miRNAs were investigated and networks were constructed (Figure 2). A total of 157 miRNAs and 286 interactions were included.

\section{Discussion}

In the present study, we tried to explore the underlying mechanisms of IS through analyzing high-throughput gene expression data and find out critical biological functions and genes. Functional enrichment analysis for the DEGs revealed 
Table 2: Characteristics of the 6 genes in the networks

\begin{tabular}{lllll}
\hline Gene ID & Symbol & Degree & Betweenness & $\begin{array}{l}\text { Clustering } \\
\text { Coefficient }\end{array}$ \\
\hline 800 & CALD1 & 6 & 3492.484 & 0.0666667 \\
1305 & COL13A1 & 6 & 772.5694 & 0.2666667 \\
3674 & ITGA2B & 13 & 26160.23 & 0.1025641 \\
3690 & ITGB3 & 36 & 103904.3 & 0.0857143 \\
4684 & NCAM1 & 12 & 66727.19 & 0.0454545 \\
7124 & TNF & 12 & 37193.15 & 0.030303 \\
\hline
\end{tabular}

that defense response, signaling pathway and apoptosis were significantly over-represented, indicating their close relationship with IS.

Inflammatory response occurs in brain tissue suffering from ischemia as a defensive mechanism ${ }^{27}$. The study by Halina et al reported that the immune response is involved in the pathological process of IS, and changes in the microenvironment of infarct zone lead to a series of activities of immune cells and immune molecules: early neutrophil invasion, activation of microglia and late macrophage invasion and activation ${ }^{28}$. Early inflammatory response is featured by neutrophils infiltration, aggregation and adhesion to vascular endothelium, and thus goes into the ischemic tissue. The aggregation of neutrophils decreases blood flow and may induce thrombosis. Moreover, activated neutrophils generate a large number of nerve toxicants, such as reactive oxygen metabolites, enzymes, protease derivatives, and cytokines like IL-1, TNF, etc. Microglia are a type of immune effector cell in the central nervous system which play a key role in the immune response ${ }^{29}$. They secrete a variety of cytokines ${ }^{30}$, which may regulate the incidence and degree of immune response. Macrophages occur in infarct zone one to three days after ischemia and reach a peak at five days, mediating the response to ischemia ${ }^{31}$. In the present study, the cytokine-mediated signaling pathway was found to be significantly over-represented in DEGs. In addition, type I the interferon-mediated signaling pathway was also overrepresented. Interferon has been associated with IS in several reports $^{32,33}$. Among many cytokines, interferon-regulatory factor-1 plays an important role in late stage of IS $^{34}$. Previous studies have reported its involvement in the immune response, cell cycle ${ }^{35}$ and apoptosis ${ }^{36}$. Studies have also indicated that ischemia can lead to apoptosis ${ }^{37}$ and the degree of apoptosis positively correlates with duration of ischemia. Apoptotic cells appear in the inner edge, i.e. the so-called "penumbra region". Immunohistochemistry confirms that most (90\%-95\%) of the apoptotic cells are nerve cells ${ }^{38}$. Conversely, inhibition of apoptosis is beneficial for protecting against IS, such as kallikrein $^{39,40}$

Interaction network analysis was performed for the top 24 DEGs and six out of them showed considerable degree, betweenness and clustering coefficient, which reflected their close relationships with other proteins and importance in the whole network. They were TNF, NCAM1, ITGB3, ITGA2B, COL13A1 and CALD1.

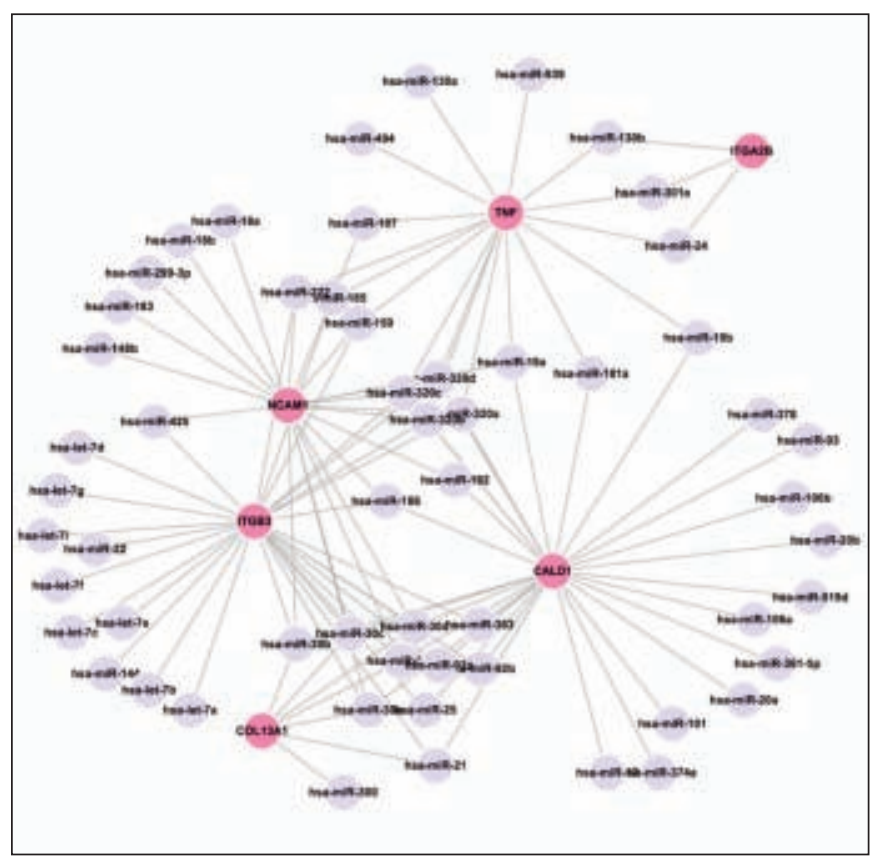

Figure 2: Regulatory networks between the 6 genes and miRNAs. Red circles represent the 6 genes and purple circles indicate miRNAs.

TNF- $\alpha$ has been reported to be closely associated with pathogenesis of $\mathrm{IS}^{41,42}$. It's a proinflammatory cytokine and involved in immune response and inflammatory reactions. Liu et al report the up-regulation of TNF- $\alpha$ mRNA and protein in ischemic neurons of rats due to focal cerebral ischemia ${ }^{42}$. Studies have shown that intraventricular injection of TNF- $\alpha$ can significantly increase the infarction size and aggravate the nerve injury, while TNF- $\alpha$ antibody can neutralize the activity of endogenous TNF- $\alpha$, which subsequently alleviates brain ischemia and reperfusion injury, and lessens infarction size ${ }^{43}$. NCAM1 is involved in cell-to-cell interactions as well as cellmatrix interactions. It is also associated with the expansion of $\mathrm{T}$ cells and dendritic cells which play an important role in immune surveillance. Yan et al find that there is a slight increase in the percentage of CD3 - CD56 + cells in the blood of stroke patients by day 7 , but this is not significantly different from controls ${ }^{44}$. ITGB3 and ITGA2B are components of integrins, which are known to participate in cell adhesion as well as cell-surface mediated signaling. The association between ITGB3 T176C polymorphism and IS has been reported ${ }^{45}$. COL13A1 is the alpha chain of one of the nonfibrillar collagens. It may serve a general function in connective tissues, but its exact function in IS is not clear. CALD 1 gene encodes a calmodulin- and actinbinding protein that plays an essential role in the regulation of smooth muscle and nonmuscle contraction ${ }^{46}$. Caldesmon and calponin are substrates for MAPK(Mitogen Activated Protein Kinase) and are associated with vasoconstriction ${ }^{47,48}$.

MicroRNAs regulating the six genes were retrieved and 178 miRNAs were obtained. Comparing with the 157 differentially expressed miRNAs reported in the study by Tan et $\mathrm{al}^{12}$, an overlap of 57 miRNAs and 108 interactions was observed, 
proving the involvements of the six genes and miRNAs in the development of IS in some degree, such as miR-103 ${ }^{49}$. Ouyang et al indicate that miR-181 regulates GRP78 and influences outcome from cerebral ischemia ${ }^{50}$. MiR-301a can regulate the expression of TNF and its downregulation helps to protect neurons from death in cerebral ischemic model ${ }^{51}$. To date, miRNAs have attracted much attention and several studies have been carried out ${ }^{49,52}$. Our findings will help to provide guidelines for future research.

In the present study, a range of DEGs were obtained which will be beneficial in advancing our understanding about the pathogenesis of IS. Six key genes were identified through network analysis. Moreover, miRNA-target gene regulatory network analysis uncovered a range of miRNAs interacting with the six genes, which could be developed into treatment tools to modulate the expression of the key genes.

\section{REFERENCES}

1. Donnan GA, Fisher M, Macleod M, Davis SM. Stroke. Lancet. 2008;371(9624):1612-23.

2. Tang $\mathrm{Y}, \mathrm{Xu} \mathrm{H}, \mathrm{Du} \mathrm{X}$, et al. Gene expression in blood changes rapidly in neutrophils and monocytes after ischemic stroke in humans: a microarray study. J Cereb Blood Flow Metab. 2006; 26(8):1089-102.

3. Pan S, Zhan X, Su X, Guo L, Lv L, Su B. Proteomic analysis of serum proteins in acute ischemic stroke patients treated with acupuncture. Exp Biol Med (Maywood). 2011;236(3):325-33.

4. Minagar A, Alexander JS, Kelley RE, Harper M, Jennings MH. Proteomic analysis of human cerebral endothelial cells activated by glutamate/MK-801: significance in ischemic stroke injury. J Mol Neurosci. 2009;38(2):182-92.

5. Jin R, Yang G, Li G. Inflammatory mechanisms in ischemic stroke: role of inflammatory cells. J Leukoc Biol. 2010;87(5):779-89.

6. Tuttolomondo A, Di Raimondo D, di Sciacca R, Pinto A, Licata G. Inflammatory cytokines in acute ischemic stroke. Curr Pharm Des. 2008;14(33):3574-89.

7. Basic Kes V, Simundic AM, Nikolac N, Topic E, Demarin V. Proinflammatory and anti-inflammatory cytokines in acute ischemic stroke and their relation to early neurological deficit and stroke outcome. Clin Biochem. 2008;41(16-17):1330-4.

8. Waje-Andreassen U, Krakenes J, Ulvestad E, et al. IL-6: an early marker for outcome in acute ischemic stroke. Acta Nerol Scand. 2005;111(6):360-5.

9. Rogaev EI. Small RNAs in human brain development and disorders. Biochemistry (Mosc). 2005;70(12):1404-7.

10. Tie J, Fan D. Big roles of microRNAs in tumorigenesis and tumor development. Histol Histopathol. 2011;26(10):1353-61.

11. Skaftnesmo KO, Prestegarden L, Micklem DR, Lorens JB. MicroRNAs in tumorigenesis. Curr Pharm Biotechnol. 2007;8 (6):320-5.

12. Tan KS, Armugam A, Sepramaniam S, et al. Expression profile of MicroRNAs in young stroke patients. PLoS One. 2009;4(11): e7689.

13. Barrett T, Edgar R. Gene expression omnibus: microarray data storage, submission, retrieval, and analysis. Methods Enzymol. 2006;411:352-69.

14. Davis S, Meltzer PS. GEOquery: a bridge between the Gene Expression Omnibus (GEO) and BioConductor. Bioinformatics. 2007;23(14):1846-7.

15. Edgar R, Domrachev M, Lash AE. Gene Expression Omnibus: NCBI gene expression and hybridization array data repository. Nucleic Acids Res. 2002;30(1):207-10.

16. Barrett T, Suzek TO, Troup DB, et al. NCBI GEO: mining millions of expression profiles--database and tools. Nucleic Acids Res. 2005;33(Database issue):D562-6.

17. Krug T, Manso H, Gouveia L, et al. Kalirin: a novel genetic risk factor for ischemic stroke. Hum Genet, 2010;127(5):513-23.
18. Montaner J, Fernandez-Cadenas I, Molina CA, et al. Poststroke Creactive protein is a powerful prognostic tool among candidates for thrombolysis. Stroke. 2006;37(5):1205-10.

19. Domingues-Montanari S, Fernandez-Cadenas I, del Rio-Espinola A, et al. The I/D polymorphism of the ACE1 gene is not associated with ischaemic stroke in Spanish individuals. Eur J Neurol. 2010;17(11):1390-2.

20. Adams HP Jr, Bendixen BH, Kappelle LJ, et al. Classification of subtype of acute ischemic stroke. Definitions for use in a multicenter clinical trial. TOAST. Trial of Org 10172 in Acute Stroke Treatment. Stroke. 1993;24(1):35-41.

21. Krug T, Gabriel JP, Taipa R, et al. TTC7B emerges as a novel risk factor for ischemic stroke through the convergence of several genome-wide approaches. J Cereb Blood Flow Metab. 2012;32 (6):1061-72.

22. Storey JD. A direct approach to false discovery rates. J R Stat Soc: Series B (Statistical Methodology). 2002;64(3):479-98.

23. Ashburner M, Ball CA, Blake JA, et al. Gene ontology: tool for the unification of biology. The Gene Ontology Consortium. Nat Genet. 2000;25(1):25-9.

24. Lewis BP, Burge CB, Bartel DP. Conserved seed pairing, often flanked by adenosines, indicates that thousands of human genes are microRNA targets. Cell. 2005;120(1):15-20.

25. Griffiths-Jones S, Grocock RJ, van Dongen S, Bateman A, Enright AJ. miRBase: microRNA sequences, targets and gene nomenclature. Nucleic Acids Res. 2006;34 (Database issue): D140-4.

26. Betel D, Wilson M, Gabow A, Marks DS, Sander C. The microRNA.org resource: targets and expression. Nucleic Acids Res. 2008;36 (Database issue):D149-53.

27. Muir KW, Tyrrell P, Sattar N, Warburton E. Inflammation and ischaemic stroke. Curr Opin Neurol. 2007;20(3):334-42.

28. Offner H, Subramanian S, Parker SM, Afentoulis ME, Vandenbark AA, Hurn PD. Experimental stroke induces massive, rapid activation of the peripheral immune system. J Cereb Blood Flow Metab. 2006;26(5):654-65.

29. Streit WJ, Conde JR, Fendrick SE, Flanary BE, Mariani CL. Role of microglia in the central nervous system's immune response. Neurol Res. 2005;27(7):685-91.

30. Hanisch UK. Microglia as a source and target of cytokines. Glia. 2002;40(2):140-55.

31. Kochanek PM, Hallenbeck JM. Polymorphonuclear leukocytes and monocytes/macrophages in the pathogenesis of cerebral ischemia and stroke. Stroke. 1992;23(9):1367-79.

32. Yilmaz G, Arumugam TV, Stokes KY, Granger DN. Role of T lymphocytes and interferon-gamma in ischemic stroke. Circulation. 2006;113(17):2105-12.

33. Liu H, Xin L, Chan BP, Teoh R, Tang BL, Tan YH. Interferon-beta administration confers a beneficial outcome in a rabbit model of thromboembolic cerebral ischemia. Neurosci Lett. 2002;327(2): 146-8.

34. Paschen W, Gissel C, Althausen S, Doutheil J. Changes in interferon-regulatory factor- 1 mRNA levels after transient ischemia in rat brain. Neuroreport. 1998;9(14):3147-51 .

35. Tanaka N, Kawakami T, Taniguchi T. Recognition DNA sequences of interferon regulatory factor 1 (IRF-1) and IRF-2, regulators of cell growth and the interferon system. Mol Cell Biol. 1993;13 (8):4531-8.

36. Horiuchi M, Yamada T, Hayashida W, Dzau VJ. Interferon regulatory factor-1 up-regulates angiotensin II type 2 receptor and induces apoptosis. J Biol Chem. 1997;272(18):11952-8.

37. Peter ME, Heufelder AE, Hengartner MO. Advances in apoptosis research. Proc Natl Acad Sci U S A. 1997;94(24):12736-7.

38. Yue X, Mehmet H, Penrice J, et al. Apoptosis and necrosis in the newborn piglet brain following transient cerebral hypoxiaischaemia. Neuropathol Appl Neurobiol. 1997;23(1):16-25.

39. Xia CF, Yin H, Borlongan CV, Chao L, Chao J. Kallikrein gene transfer protects against ischemic stroke by promoting glial cell migration and inhibiting apoptosis. Hypertension. 2004;43(2): 452-9.

40. Xia CF, Yin H, Yao YY, Borlongan CV, Chao L, Chao J. Kallikrein protects against ischemic stroke by inhibiting apoptosis and 
inflammation and promoting angiogenesis and neurogenesis. Hum Gene Ther. 2006;17(2):206-19.

41. Zaremba J, Losy J. Early TNF-alpha levels correlate with ischaemic stroke severity. Acta Neurol Scand. 2001;104(5):288-95.

42. Liu T, Clark RK, McDonnell PC, et al. Tumor necrosis factor-alpha expression in ischemic neurons. Stroke. 1994;25(7):1481-8.

43. Belosjorow S, Bolle I, Duschin A, Heusch G, Schulz R. TNF-alpha antibodies are as effective as ischemic preconditioning in reducing infarct size in rabbits. Am J Physiol Heart Circ Physiol. 2003;284(3):H927-30.

44. Yan J, Greer JM, Etherington K, et al. Immune activation in the peripheral blood of patients with acute ischemic stroke. J Neuroimmunol. 2009;206(1-2):112-7.

45. Wei YS, Lan Y, Liu YG, Meng LQ, Xu QQ, Xie HY. [Association of the integrin gene polymorphisms with ischemic stroke and plasma lipid levels]. Zhonghua Yi Xue Yi Chuan Xue Za Zhi. 2009;26(2):211-5.

46. Wang CL. Caldesmon and the regulation of cytoskeletal functions. Adv Exp Med Biol. 2008; 644:250-72.
47. Suzuki H, Hasegawa Y, Kanamaru K, Zhang JH. Mitogen-activated protein kinases in cerebral vasospasm after subarachnoid hemorrhage: a review. Acta Neurochir Suppl. 2011;110(Pt 1): 133-9.

48. Jiang Q, Huang R, Cai S, Wang CL. Caldesmon regulates the motility of vascular smooth muscle cells by modulating the actin cytoskeleton stability. J Biomed Sci. 2010;17(6):6.

49. Wang Y, Yang GY. MicroRNAs in cerebral ischemia. Stroke Res Treat. 2013;2013:276540.

50. Ouyang YB, Lu Y, Yue S, et al. miR-181 regulates GRP78 and influences outcome from cerebral ischemia in vitro and in vivo. Neurobiol Dis. 2012;45(1):555-63.

51. Kaur P, Armugam A, Jeyaseelan K. MicroRNAs in Neurotoxicity. J Toxicol. 2012;2012:870150.

52. Ouyang YB, Stary CM, Yang GY, Giffard R. microRNAs: innovative targets for cerebral ischemia and stroke. Curr Drug Targets. 2013;14(1):90-101. 